\title{
Gastric Invasive Micropapillary Carcinoma with Intestinal Phenotypes Harboring a TP53 R175H Mutation
}

\author{
Hideyuki Ikeda ${ }^{\mathrm{a}}$ Takehiro Kukitsu $^{\mathrm{b}}$ Wataru Johmen $^{\mathrm{b}, \mathrm{d}}$ \\ Hajime Nakamura $^{b}$ Natumi Yamauchi ${ }^{b}$ Kazuma Ishikawa ${ }^{b}$ \\ Takashi Saikawa $^{\mathrm{b}}$ Sayaka Noda ${ }^{\mathrm{b}}$ Tadanori Saitoh $^{\mathrm{b}}$ Yoshinori Ueno ${ }^{\mathrm{b}}$ \\ Yuuya Noda ${ }^{c}$ Sayuki Yamazaki ${ }^{c}$ Yoshikazu Kuroda ${ }^{c}$ \\ Susumu Koshiko ${ }^{c, e}$ Yutaka Sasagawa $^{b}$ \\ Divisions of a Surgical Pathology, ${ }^{b}$ Internal Medicine and ' Surgery, Rumoi City Hospital, \\ Rumoi, ${ }^{\mathrm{d}}$ Department of Medical Oncology and Hematology, Sapporo Medical University

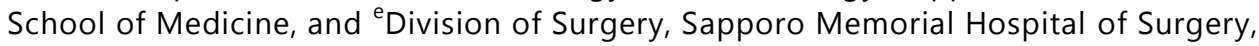 \\ Sapporo, Japan
}

\section{Key Words}

Gastric cancer - Invasive micropapillary carcinoma - Intestinal phenotypes · CD10 - TP53 ·

$\mathrm{R} 175 \mathrm{H}$

\begin{abstract}
We report a case of gastric invasive micropapillary carcinoma (IMPC) in an 86-year-old female patient. She was admitted to our hospital with a chief complaint of bloody emesis. Upper gastrointestinal endoscopy found a gastric adenocarcinoma at the antrum. The biopsy specimens showed moderately differentiated adenocarcinoma with invasive small tumor nests. Distal gastrectomy with systematic lymph node dissection demonstrated that the tumor had IMPC through a pathological examination. Despite the depth of tumor invasion (the submucosa), extensive lymph node metastases were observed. Anti-D2-40 immunostaining revealed numerous infiltrating tumor cell nests in the lymphatic vessels, which could explain subsequent multiple lymph node metastases. The adenocarcinoma showed intestinal phenotypes by several immunohistochemical studies. One of these antibodies (CD10) clearly demonstrated the inverted apical-basal (inside-out) pattern of IMPC, whereas it showed an ordinary pattern in intestinal metaplasia adjacent to the tumor. Furthermore, genetic analysis by direct sequencing revealed a point mutation in the exon 5 of TP53 in the
\end{abstract}


Ikeda et al:: Gastric Invasive Micropapillary Carcinoma with Intestinal Phenotypes Harboring a TP53 R175H Mutation

tumor. The mutation presumably harbors a missense mutation from Arg to His at the codon $175(\mathrm{R} 175 \mathrm{H}) . \mathrm{R} 175 \mathrm{H}$ has been previously described as a 'gain-of-function' mutation with a high invasive or metastatic potential in several types of cancers. In summary, this is one of the first reported cases of gastric IMPC with intestinal phenotypes harboring a TP53 R175H mutation in the literature.

(C) 2014 S. Karger AG, Basel

\section{Introduction}

Invasive micropapillary carcinoma (IMPC) was initially described as a histological variant of breast cancer, characterized as a carcinoma composed of little clumps of tumor cells lying within clear spaces separated by a fibrocollagenous or delicate fibrovascular stroma [1]. Since then, it has been reported in various kinds of cancers derived from breast, urinary bladder, endometrium, lung, salivary gland [2] and in cancers of the gastrointestinal tract [3]. Although reports of gastric cancers with IMPC are very limited [4, 5], a high frequency of lymphovascular invasions and metastases in lymph nodes have been described recently $[3$, $6,7]$. We report a case of gastric IMPC with intestinal phenotypes. Upon genetic analysis, we have found a TP53 R175H mutation in the tumor. To the best of our knowledge, this is the first report of gastric IMPC with a TP53 R175H mutation that has a high invasive or metastatic potential in the literature. The patient's family member gave written informed consent for this report, and the identity of the patient has been strictly protected.

\section{Case Presentation}

An 86-year-old Japanese female patient was admitted to our hospital with a chief complaint of bloody emesis. She had a flat abdomen and did not complain of any abdominal pain. Present status on admission was the following: body height: $151 \mathrm{~cm}$, body weight: $71 \mathrm{~kg}$, body temperature: $36{ }^{\circ} \mathrm{C}$, blood pressure: $172 / 75 \mathrm{~mm} \mathrm{Hg}$, pulse: $88 \mathrm{bpm}$, palpebral conjunctiva: anemic, bulbar conjunctiva: not icteric. Laboratory data on admission noted $\mathrm{Hb}$ $6.8 \mathrm{~g} / \mathrm{dl}$, CEA $80.1 \mathrm{ng} / \mathrm{ml}$ and CA19-9 $74.1 \mathrm{U} / \mathrm{ml}$. No specific past and family history was recorded. Upper gastrointestinal endoscopy found a gastric tumor at the antrum. After endoscopic hemostasis, endoscopic biopsy was done; the diagnosis was moderately differentiated adenocarcinoma with invasive small tumor nests (fig. 1a). Systemic examination, including endoscopic ultrasonography and computerized tomography, determined that the preoperative stage of the patient was T2 N2 M0; clinical stage IIB (UICC; 7th edition). She underwent distal gastrectomy with systematic lymph node dissection and additional cholecystectomy, followed by Billroth I reconstruction.

Surgically resected specimens were subjected to pathological evaluation. The specimen resected by distal gastrectomy (approx. $16.7 \times 12.8 \mathrm{~cm}$ ) demonstrated that a slightly elevated lesion $(2.6 \times 2.2 \mathrm{~cm})$ with ulceration $(1.6 \times 1.6 \mathrm{~cm})$ was present at the lesser curvature of the antrum (fig. 1b). Histopathologically, moderately differentiated adenocarcinoma with small tumor nests was located adjacent to or overlapped with well-differentiated adenocarcinoma in serial sections of the entire lesion (fig. 1c). The small tumor nests that were surrounded by lacunar-like clear space and dense fibrous stroma showed an inverted apical-basal (inside-out) pattern (fig. 2a). No fibrovascular core was present in the small nests, which was confirmed by anti-CD34 immunostaining (data not shown). Thus, they represented a typical IMPC. The IMPC components occupied approximately $50 \%$ of the total tumor mass (fig. 1c). Immunostaining with anti-EMA monoclonal antibody (mAb) showed 
Ikeda et al:: Gastric Invasive Micropapillary Carcinoma with Intestinal Phenotypes Harboring a TP53 R175H Mutation

the inside-out pattern; however, simultaneous cytoplasmic staining made the pattern unclear (data not shown). On the other hand, anti-CD10 mAb clearly demonstrated the distinct linear positivity on the stroma-facing surfaces of the small nests (fig. 2b). This reversed polarity provides a marked contrast to intestinal metaplasia that shows an ordinary apical-basal pattern adjacent to the tumor (fig. 2b, inset). IMPC showed an infiltrative growth into the submucosa (depth: approx. $2 \mathrm{~mm}$ ) but no invasion into the propria muscle. More importantly, lymphovascular invasions were strongly suspected. AntiD2-40 immunostaining that reacts against normal lymphatic vessels revealed numerous infiltrating tumor nests in the vessels (fig. 2c). Moreover, systematic regional lymph node dissection demonstrated extensive lymph node metastases ( 9 positives out of 20 examined). Taken together, the pathological diagnosis of the tumor was a relatively early gastric cancer with IMPC and multiple lymph node metastases [T1b2 N3a M0; pathological stage IIB (UICC, 7 th edition)]. The entire tumoral lesion was situated on the boundary between the fundic glands without atrophy and pyloric glands with intestinal metaplasia (fig. 1c). Helicobacter pylori infection was found positive by microscopy. No specific finding was observed in the resected gall bladder.

As noted above, the tumor was positive by immunohistochemistry for CD10 and EMA. Further immunohistochemical analysis was performed for immunophenotyping of the tumor. All antibodies used in this study are summarized in table 1. In general, gastric adenocarcinomas with typical gastric phenotypes are negative for MUC2, CK20 and CD10, whereas they are positive for both MUC5AC and MUC6. In our case, the tumor was positive for MUC2, CDX2 and CK20, although either partially or very faintly. On the other hand, it was negative for both MUC5AC and MUC6; therefore, it is very likely that the tumor was a gastric adenocarcinoma with intestinal phenotypes (table 2).

Both anti-Ki-67 and p53 mAbs strongly stained the nuclei of the tumor cells (data not shown). In order to know the genetic status of TP53, cancer-cell rich portions were excised from paraffin-embedded tissue sections. Genomic DNA was extracted by standard procedures. Purified DNA was amplified by PCR and the products were directly sequenced according to the IARC protocols with slight modifications [8]. All TP53-specific primers for both PCR amplification and sequencing are summarized in table 3. A representative electropherogram indicated a point mutation of the G to A transition in the exon 5 (fig. 3). This transition presumably harbors a missense mutation from Arg to His at the codon 175 (R175H). Sequence analysis in the opposite direction was also performed, and the mutation was confirmed (data not shown).

The postoperative state of the patient was quite favorable. No chemotherapy was performed in consideration of her age. The patient has been alive for 4 years without any episode of tumor relapse or distant metastasis.

\section{Discussion}

Thus far, several Asian groups have exclusively reported gastric IMPC in the literature $[4-7,9]$. This is probably because either gastric IMPC has closely related to environmental factors such as dietary habits of a high salt intake or dominant $H$. pylori infection throughout the area. Otherwise, the concept of gastric IMPC itself may not have been widely accepted in western countries. Owing to those pioneering reports, however, we understand that gastric IMPC is a rare but distinct variant of adenocarcinoma that shares histologic and immunohistochemical features with other kinds of cancers. In gastric cancers with IMPC, a high frequency of lymphovascular invasions and metastases in lymph nodes has been described 
Ikeda et al:: Gastric Invasive Micropapillary Carcinoma with Intestinal Phenotypes Harboring a TP53 R175H Mutation

$[3,6,7]$. Furthermore, it has been known that tumors with IMPC may undergo poor prognosis because of frequent metastasis, especially at an early stage [5]. In our case, despite the depth of tumor invasion (the submucosa), extensive lymphovascular invasions and multiple lymph node metastases were observed. Several molecules, such as MUC2, betacatenin and CD44v6, have been discussed in the light of cell shape, IMPC polarity and grade of malignancy $[3,5]$. Nevertheless, molecular analysis has never been conducted in gastric IMPC; factors that are responsible for the frequent lymphovascular invasions and metastases remain unclear. We have found a TP53 R175H mutation in the tumor. According to the IARC TP53 database (release 17), out of a total of 29,711 entries, 1,215 cases of R175H mutation have been registered, which is the largest number of all mutations in the database. Among them, 67 cases of R175H mutation in gastric cancers have been reported [10]. Mutated TP53 function of $\mathrm{R} 175 \mathrm{H}$ has been especially described as a 'gain-of-function' mutation, in addition to the loss of tumor suppression function and dominant negative effects. In our case, it is likely that R175H was expressed heterologously with the wild-type allele (fig. 3). Therefore, the mutated p53 may have exerted a gain of function as well as tumor suppression function and dominant negative effects in the tumor cells. In several human tumor models, the high invasive or metastatic mechanisms of the p53 R175H mutation have been investigated. In one example, the invasive phenotype of endometrial cancer cells with $\mathrm{R} 175 \mathrm{H}$ was discussed in the context of the EGFR/PI3K/AKT pathways [11]. Similarly, an activation of c-Met receptor tyrosine kinase for tumor invasion was reported upon $\mathrm{R} 175 \mathrm{H}$ overexpression in esophageal squamous cell carcinoma [12]. Moreover, E-cadherin was focused on a breast cancer cell line with R175H mutation in terms of cell shape and cell growth [13]. This is particularly interesting because the loss of E-cadherin expression is strongly associated with the invasive nature of breast lobular carcinomas. We stained the gastric tumor cells with anti-E-cadherin antibody; however, no significant differences were observed between normal cells and tumor cells with or without IMPC (data not shown). We have conducted the sameTP53 mutational analyses in several cases of gastric and colorectal cancers with IMPC phenotypes. We have found distinct TP53 mutations in addition to this report; however, no identical mutation has been found [Ikeda, unpubl. data: G154P in a gastric cancer; E258K in a rectal cancer]. In colon cancers with IMPC, similar studies have been performed, and a higher proportion of TP53 alteration was reported [14]. In this report, however, no specific TP53 mutation was described.

Anti-EMA/MUC1 staining is commonly used to show the inverted pattern of IMPC [9, 14]. CD10 is reported to be useful for depicting the pattern of sigmoid colon cancer [15]. In gastric cancers, however, CD10 was not effective in demonstrating IMPC with obvious gastric phenotypes $[4,9]$. In our case, EMA could show the positive inside-out pattern of the tumor cells; however, simultaneous cytoplasmic staining made the pattern unclear. CD10 is expressed on normal small intestinal brush border (fig. $2 \mathrm{~b}$, inset). Thus, it is quite reasonable for CD10 to indicate the inside-out pattern of IMPC of gastric adenocarcinoma with intestinal phenotypes. CD10 was also positive for metastatic tumor in the lymph nodes while keeping the reversed polarity. In this situation, we recognized the tumor nests as well as resident lymphoid follicles that were positive for CD10 in the background.

In summary, the present report is one of the first reported cases of gastric IMPC with intestinal phenotypes harboring a TP53 $\mathrm{R} 175 \mathrm{H}$ mutation in the literature. In order to generalize whether gastric IMPC with a TP53 R175H mutation will show multiple lymph node metastases at an early stage of the tumor, accumulation of similar clinical cases and further analysis is necessary in the future. 
Ikeda et al:: Gastric Invasive Micropapillary Carcinoma with Intestinal Phenotypes Harboring a TP53 R175H Mutation

\section{Acknowledgments}

We thank Dr. Mitsuhiro Tada for his critical reading of the manuscript. We also thank Takayuki Ohnishi, Ken-ichi Kimura and Masayo Itoh for their excellent technical assistance.

\section{Disclosure Statement}

The authors have no conflicts of interest relevant for this report.

\section{References}

1 Siriaunkgul S, Tavassoli FA: Invasive micropapillary carcinoma of the breast. Mod Pathol 1993;6:660-662.

-2 Nassar H: Carcinomas with micropapillary morphology: clinical significance and current concepts. Adv Anat Pathol 2004;11:297-303.

-3 Guzińska-Ustymowicz K, Niewiarowska K, Pryczynicz A: Invasive micropapillary carcinoma: a distinct type of adenocarcinomas in the gastrointestinal tract. World J Gastroenterol 2014;20:4597-4606.

-4 Roh JH, Srivastava A, Lauwers GY, et al: Micropapillary carcinoma of stomach: a clinicopathologic and immunohistochemical study of 11 cases. Am J Surg Pathol 2010;34:1139-1146.

$\checkmark 5$ Eom D-W, Kang GH, Han SH, et al: Gastric micropapillary carcinoma: a distinct subtype with a significantly worse prognosis in TNM stages I and II. Am J Surg Pathol 2011;35:84-91.

-6 Ushiku T, Matsusaka K, Iwasaki Y, et al: Gastric carcinoma with invasive micropapillary pattern and its association with lymph node metastasis. Histopathology 2011;59:1081-1089.

-7 Fujita T, Gotohda N, Kato Y, et al: Clinicopathological features of stomach cancer with invasive micropapillary component. Gastric Cancer 2012;15:179-187.

8 IARC Protocol, 2010 update: Detection of TP53 mutations by direct sequencing. http://wwwp53.iarc.fr/Download/TP53_DirectSequencing_IARC.pdf.

-9 Ohtsuki Y, Kuroda N, Yunoki S, et al: Immunohistochemical analysis of invasive micropapillary carcinoma pattern in four cases of gastric cancer. Med Mol Morphol 2013;46:114-121.

-10 Petitjean A, Mathe E, Kato S, et al: Impact of mutant p53 functional properties on TP53 mutation patterns and tumor phenotype: lessons from recent developments in the IARC TP53 database. Hum Mutat 2007;28:622-629.

11 Dong P, Xu Z, Jia N, et al: Elevated expression of p53 gain-of-function mutation R175H in endometrial cancer cells can increase the invasive phenotypes by activation of the EGFR/PI3K/AKT pathway. Mol Cancer 2009;8:103.

12 Grugan KD, Vega ME, Wong GS, et al: A common p53 mutation (R175H) activates c-Met receptor tyrosine kinase to enhance tumor cell invasion. Cancer Biol Ther 2013;14:853-859.

-13 Rieber M, Strasberg Rieber M: DN-R175H p53 mutation is more effective than p53 interference in inducing epithelial disorganization and activation of proliferation signals in human carcinoma cells: role of $\mathrm{E}$ cadherin. Int J Cancer 2009;125:1604-1612.

14 Verdú M, Román R, Calvo M, et al: Clinicopathological and molecular characterization of colorectal micropapillary carcinoma. Mod Pathol 2011;24:729-738.

15 Wen P, Xu Y, Frankel WL, et al: Invasive micropapillary carcinoma of the sigmoid colon: distinct morphology and aggressive behavior. Int J Clin Exp Pathol 2008;1:457-460. 
Oncology

DOI: $10.1159 / 000367583$

(C) 2014 S. Karger AG, Base

www.karger.com/cro

Ikeda et al.: Gastric Invasive Micropapillary Carcinoma with Intestinal Phenotypes Harboring a TP53 R175H Mutation

Table 1. Primary antibodies used in the study

\begin{tabular}{lllll}
\hline Product name & Dilution & Clone & Pretreatment & Manufacturer \\
\hline CK7 & $1: 50$ & OV-TV12/30 & AC & DAKO \\
CK20 & $1: 25$ & Ks20.8 & AC & DAKO \\
HMW keratin & $1: 50$ & 34 bE12 & AC & DAKO \\
EMA & $1: 100$ & E29 & AC & DAKO \\
CD10 & $1: 50$ & $56 C 6$ & AC & DAKO \\
MUC2 & $1: 50$ & CCP58 & WB & Novocastra \\
CDX2 & $1: 50$ & CDX2-88 & AC & BioGenex \\
MUC5AC & $1: 50$ & CLH2 & WB & Novocastra \\
MUC6 & $1: 100$ & CLH5 & WB & Novocastra \\
D2-40 & $1: 50$ & D2-40 & AC & DAKO \\
CD34 & $1: 50$ & QBEnd10 & AC & DAKO \\
E-cadherin & $1: 100$ & NHC-38 & AC & DAKO \\
Ki-67 & $1: 50$ & MIB-I & AC & DAKO \\
P53 & $1: 50$ & D0-7 & AC & DAKO \\
\hline
\end{tabular}

Secondary antibody and detection system: DAKO EnVision ${ }^{\mathrm{TM}}$. HMW keratin = high-molecular-weight keratin; $\mathrm{AC}=$ autoclave, $120^{\circ} \mathrm{C}, 20 \mathrm{~min} ; \mathrm{WB}=$ water bath, $100^{\circ} \mathrm{C}, 30 \mathrm{~min}$.

Table 2. Gastric adenocarcinoma with intestinal phenotypes

$\begin{array}{ll}\text { CK7 } & + \\ \text { CK20 } & +/- \\ \text { HMW keratin } & - \\ \text { CD10 } & +/- \\ \text { CDX2 } & -/+ \\ \text { MUC2 } & -/+ \\ \text { MUC5AC } & - \\ \text { MUC6 } & -\end{array}$

See text for gastric adenocarcinoma with ordinary gastric phenotypes. $+=$ Positive; +/- = definite but segmentally or partially positive; $-/+=$ very faintly positive; - = negative; HMW keratin = high-molecularweight keratin: clone $34 \mathrm{bE} 12$. 
Ikeda et al.: Gastric Invasive Micropapillary Carcinoma with Intestinal Phenotypes Harboring a TP53 R175H Mutation

Table 3. TP53-specific primers used in the study

\begin{tabular}{llll}
\hline & Forward primer & Reverse primer & $\begin{array}{l}\text { Expected } \\
\text { length }\end{array}$ \\
\hline $\begin{array}{l}\text { For PCR amplification } \\
\text { Exon } 5\end{array}$ & & & \\
Exon 6 & $5^{\prime}$-TATCTGTTCACTTGTGCCCT-3' & $5^{\prime}$-CAGACCTAAGAGCAATCAGT-3' & $324 \mathrm{bp}$ \\
Exon 7 & $5^{\prime}$-ACGACAGGGCTGGTTGCCCA-3' & $5^{\prime}$-CCCTACTGCTCACCCGGAGG-3' & $248 \mathrm{bp}$ \\
Exon 8 & $5^{\prime}$-GCGACAGAGCGAGATTCCAT-3' & $5^{\prime}$-TCAGCGGCAAGCAGAGGCTG-3' & $282 \mathrm{bp}$ \\
\hline For sequencing & $5^{\prime}$-TGGGACAGGTAGGACCTGAT-3' & $5^{\prime}$-GTGAATCTGAGGCATAACTG-3' & $260 \mathrm{bp}$ \\
Exon 5 & & & \\
Exon 6 & $5^{\prime}$-TATCTGTTCACTTGTGCCCT-3' & $5^{\prime}$-CAGACCTAAGAGCAATCAGT-3' & \\
Exon 7 & $5^{\prime}$-ACGACAGGGCTGGTTGCCCA-3' & $5^{\prime}$-GAGGGCCACTGACAACCACC-3' & \\
Exon 8 & $5^{\prime}$-CCTGCTTGCCACAGGTCTCC-3' & $5^{\prime}$-TCAGCGGCAAGCAGAGGCTG-3' & \\
\hline
\end{tabular}

All sequences are derived from the reference sequence with an accession number: X54156. bp = Base pairs. 


\section{Case Reports in Oncology}

\begin{tabular}{l|l}
\hline Case Rep Oncol 2014;7:611-620 \\
\hline DOI: $10.1159 / 000367583$ & $\begin{array}{l}\text { C 2014 S. Karger AG, Basel } \\
\text { www.karger.com/cro }\end{array}$ \\
\hline
\end{tabular}

Ikeda et al.: Gastric Invasive Micropapillary Carcinoma with Intestinal Phenotypes Harboring a TP53 R175H Mutation
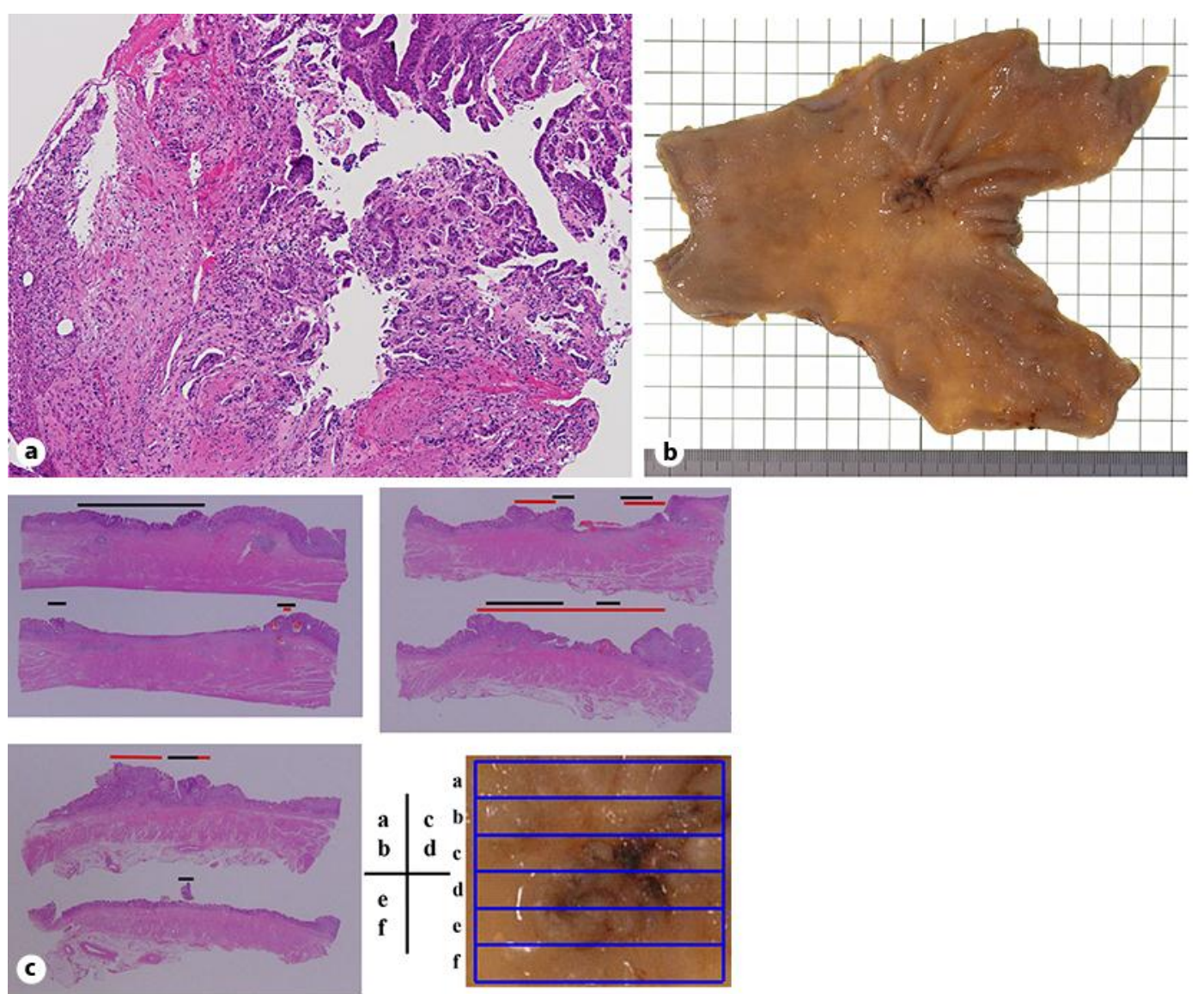

Fig. 1. a A biopsy specimen in endoscopy. Moderately differentiated adenocarcinoma with invasive small tumor nests. Original magnification is $\times 40$, HE stain. $\mathbf{b}$ A surgical specimen by distal gastrectomy (approx. $16.7 \times 12.8 \mathrm{~cm})$. A slightly elevated lesion $(2.6 \times 2.2 \mathrm{~cm})$ with ulceration $(1.6 \times 1.6 \mathrm{~cm})$ is present at the lesser curvature of the antrum. $\mathrm{c}$ Semi-macro images of the entire lesion in serial sections from a through $\mathrm{f}$ (HE stain). Red lines indicate the presence of a moderately differentiated adenocarcinoma with IMPC. The well-differentiated adenocarcinoma is indicated by black lines. 


\section{Case Reports in Oncology}

\begin{tabular}{l|l}
\hline Case Rep Oncol 2014;7:611-620 \\
\hline DOI: $10.1159 / 000367583$ & $\begin{array}{l}\text { C 2014 S. Karger AG, Basel } \\
\text { www.karger.com/cro }\end{array}$ \\
\hline
\end{tabular}

Ikeda et al.: Gastric Invasive Micropapillary Carcinoma with Intestinal Phenotypes Harboring a TP53 R175H Mutation
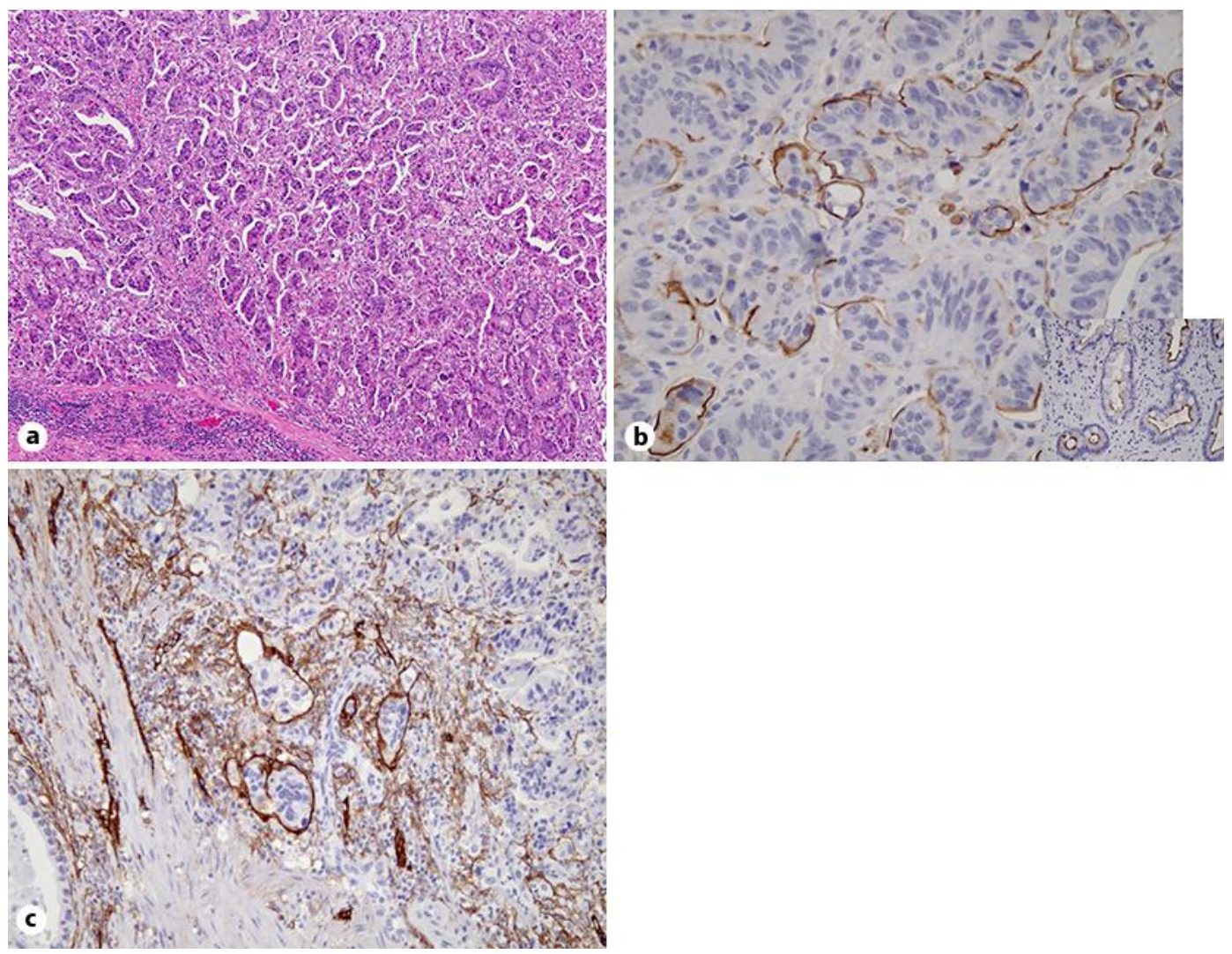

Fig. 2. a Small nests of tumor cells in the mucosa showing the typical inverted apical-basal (inside-out) pattern of IMPC (fig. 1c/section d, right part). Original magnification is $\times 40$, HE stain. b Anti-CD10 immunostaining shows the inside-out pattern of IMPC. Original magnification is $\times 200$. Inset Anti-CD10 immunostaining of nontumoral intestinal metaplasia adjacent to the tumor shows an ordinary apicalbasal pattern. Original magnification is $\times 200$. c Anti-D2-40 immunostaining shows lymphatic vessels containing numerous infiltrating tumor cells with IMPC (the same as b). Original magnification is $\times 100$. 
Ikeda et al:: Gastric Invasive Micropapillary Carcinoma with Intestinal Phenotypes Harboring a TP53 R175H Mutation

TP53 exon 5 codon 175 CGC (Arg) to CAC (His)

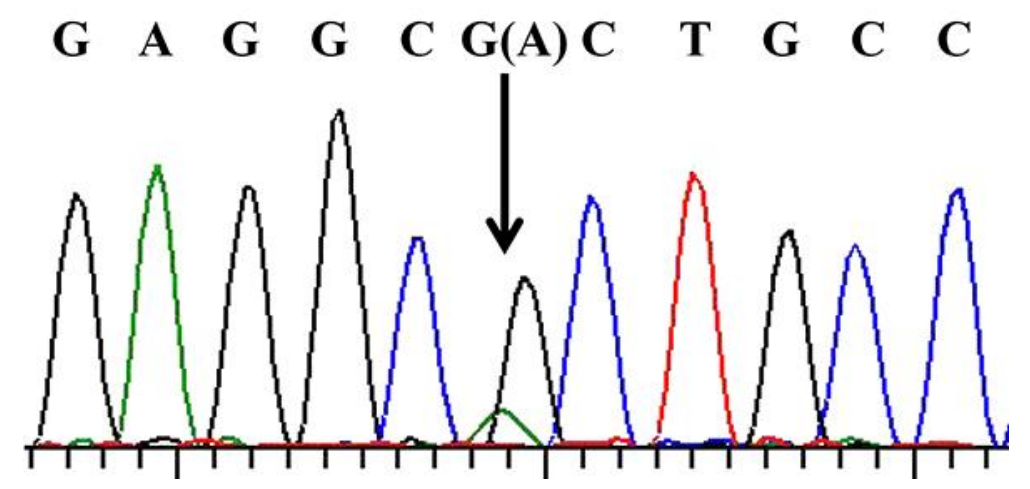

Fig. 3. A representative electropherogram by direct sequencing of TP53. A point mutation of the G to A transition is indicated in exon 5, which presumably harbors a missense mutation from Arg to His at codon 175 (R175H). 